\title{
Vitreous humor
}

National Diabetes Information Clearinghouse (NDIC)

\section{Definitions}

Retina

Defined by National Diabetes Information Clearinghouse (NDIC)

\section{Source}

National Diabetes Information Clearinghouse (U.S.). (2009). The diabetes dictionary. [Bethesda, Md.]: U.S. Dept. of Health and Human Services, National Institutes of Health, National Institute of Diabetes and Digestive and Kidney Diseases, National Diabetes Information Clearinghouse.

The clear gel that lies behind the eye's lens and in front of the retina. 